\title{
Review Article \\ Critical Review on Effect of 5G Technology on Covid-19 and Human Health Issues
}

\author{
B. S. Sedani ${ }^{1 *}$, N. A. Kotak $^{1}$, K. R. Borisagar ${ }^{3}$ \\ ${ }^{1}$ Department of Electronics and Communication Engineering, L. D. College of Engineering, \\ Ahmedabad, India \\ ${ }^{3}$ Graduate School of Engineering \& Technology, Gujarat Technological University, Ahmedabad, \\ India
}

Received 3 October 2020, accepted in final revised form 5 February 2021

\begin{abstract}
This analytic research paper discusses the effects of emerging futuristic 5th Generation (5G) cellular technology over human health. The technology is growing with three major advantages of high speed, lower latency and to connect more devices at the same time. In the recent pandemic event of Covid-19, many believe that for the achievement of high speed in $5 \mathrm{G}$, it will be required to have high power and this high power will decrease the immune system in short term. But actually, the reality doesn't support the above condition. This paper defines how the speed will increase with the help of low bit error rate and also through different useful techniques thereby not just by increasing the power. In addition of high-frequency $5 \mathrm{G}$ radiation to an already complex mix of lower frequencies, the implementation will contribute to a negative public health outcome both from physical and mental health perspectives to a certain extent.
\end{abstract}

Keywords: 5G wireless technology; Covid-19; Massive MIMO; Beam forming; Health effects.

(C) 2021 JSR Publications. ISSN: 2070-0237 (Print); 2070-0245 (Online). All rights reserved. doi: http://dx.doi.org/10.3329/jsr.v13i2.49514 J. Sci. Res. 13 (2), 695-705 (2021)

\section{Introduction}

$5 \mathrm{G}$ is one of the most lucrative innovations approved out by Federal Communications Commission (FCC) which will be a new faster and smooth telecommunication technology. The speed in terms of Gbps and the efficiency anticipated by 5 th Generation technology would be very high. The need of 5G technology increases day by day due to huge increment in no. of users, real time data calculation and receiver complexity, etc. Nowadays $4 \mathrm{G}$ is the technology adapted by almost all over globe but as a demand of number of users and devices is going to increase day by day; there will be too many users in $4 \mathrm{G}$ spectrum. To accommodate the increased demand, the implementation of $5 \mathrm{G}$

\footnotetext{
*Corresponding author: bhavin_s_sedani@yahoo.com
} 
technology must be geared up. In India, the implementation of $5 \mathrm{G}$ will become available in 2022 [1]. Basically, 5G bands have been defined in terms of three band spectrum.

Low band spectrum-Here the frequency band is less than $1 \mathrm{GHz}$; the band is generally used for the LTE applications. It offers high coverage but the data speed will be limited to 100 Mbps [2].

Mid band spectrum - It provides faster speed and lower latency as compare to low band spectrum but it fails to penetrate the building as compare to low band spectrum that's why the use of massive MIMO and small cell may improve the penetration and coverage area of mid band. Maximum achievable data speed will be up to $1 \mathrm{Gbps}$.

High band spectrum - This band has the highest performance among all 5G spectrum bands. It can offer speed up to $100 \mathrm{Gbps}$ but it's often known as mm Wave band which has low coverage area. To overcome this problem, beam forming technique may be used. Different 5G spectrums and their applications are defined in Table 1.

\section{5G Effects on Covid-19}

Corona virus disease (COVID-19) is an infectious disease caused by a newly discovered corona virus. The virus that causes COVID-19 is mainly transmitted through droplets generated when an infected person coughs, sneezes, or exhales. These droplets are too heavy to hang in the air, and quickly fall on floors or surfaces.

Table 1. Different 5G spectrums and their applications.

\begin{tabular}{|c|c|c|}
\hline Frequency range & Use & Description \\
\hline$<1 \mathrm{GHz}$ & IoT applications, data coverage & $\begin{array}{l}\text { Longer range coverage, less } \\
\text { costly infrastructure }\end{array}$ \\
\hline $1-6 \mathrm{GHz}$ & $\begin{array}{l}\text { Data coverage, IoT application, } \\
\text { moderate capacity for data transfer }\end{array}$ & $\begin{array}{l}\text { More spectrum available, } \\
\text { shorter range }\end{array}$ \\
\hline$>6 \mathrm{GHz}$ & Large data transfer with high speed & $\begin{array}{l}\text { Allow high speed data for low } \\
\text { latency time }\end{array}$ \\
\hline
\end{tabular}

Due to recent Covid-19 pandemic, certain incidents took place in England in which the transmission towers of $5 \mathrm{G}$ had been burnt down due to spreading of rumor that high power transmission for 5G technology is the reason behind it. Moreover, even today, some communities in the world still believe in some theory that $5 \mathrm{G}$ technology is culprit behind some infections. They believe that to increase speed and bandwidth of system, transmission of high power is required and this high power will affect our short-term immune system which in turn will cause more infections of Covid-19. This theory is based on the radiation system and the immune system of human body in which it has been said for years that the signal from mobile radiation harms the body for a long time and there is a possibility of cancer, tumor etc. However, these issues are discussed in next upcoming sections of this paper. But in the case of spreading of Corona virus, as on date, no cure has been found for it, only the immune system of the human body is important. Now if we talk about $5 \mathrm{G}$ technologies, the millimeter signal of such high frequency does 
not have enough energy to break the muscles and cells of our body. Also, the key point is there are some power restrictions applied by FCC, due to this transmission power has some limit in 5G technology. So, in the current situation, it is not at all the fact that in a very short period of time, $5 \mathrm{G}$ radiation will weaken the immune system of a human body which will lead to increasing effects of Covid-19. It is bit clear that Covid-19 cannot be caused by $5 \mathrm{G}$, in fact certain areas of world are also there where there is no $5 \mathrm{G}$ but Covid19 is there. So rather just making $5 \mathrm{G}$ system to be the entire culprit, one might consider that a weakened immune system might be a co-factor caused by RF-radiation from existing transmission systems (3G, 4G, 4G+, etc.). Another key aspect in 5G system regarding increase in the speed and bandwidth of system, massive MIMO which is space diversity technique will be utilized and also the bit error rate is decreased with the help of different types of coders to anticipate speed and bandwidth utilities.

As this is the cellular system that means the smallest and the base unit of whole architecture is single cell, in 5G technology the basic cells are also divided in smaller cells so that the transmission power will also get decrease for the given coverage area. Hence it can be stated that in $5 \mathrm{G}$, to increase speed and bandwidth that to increase overall system capacity, power is not going to be increased beyond certain limit, rather it is lesser in the smaller size cell than what it is expected. So, the cause of health hazard is not the increment in power to increase capacity.

Actually, there will be health hazard due to Beam forming technique of $5 \mathrm{G}$ system where in power is concentrated to a particular point rather than huge amount of power. This highly concentrated power can be harmful for human health but it is long time process. A sudden decrease in human immune system will not be caused by $5 \mathrm{G}$ towers.

One more fact can support the above discussed analysis. It can be seen and verified by looking at some of the countries like Iran, wherein still 5G services have not been implemented yet but still the people over there are affected by Covid-19.

As whole it can be verified that there are many techniques lying in $5 \mathrm{G}$ from which required speed and coverage can be increased rather than just power increase. Some of the possible techniques are listed below in the next section of article.

\section{Key Enabling Technologies in 5G}

\subsection{Small cell architecture}

In $5 \mathrm{G}$ spectrum, the coverage area is very small for high band and mid band spectrum ranges so the use of old clustering techniques will not be useful in 5G [2-4].

In currently implemented wireless cellular system, the area is divided in small cells, the group of cells is known as cluster and in every cell, there is large high-power cell tower to broadcast signal, over long distance.

Whereas in 5G system with high band spectrum, there will be millimeter ( $\mathrm{mm}$ ) wave so it can travel only a short distance and also not able to travel through obstacles, which means if devices are behind any obstacles, they lose the signal. But dividing the cells in small cell networks will solve that problem. Using thousands of small towers as a base 
station, all the base are close together, small cell will be able to transmit signal around the obstacles easily. As user moves around the obstacles, the device, get automatically switched from one nearest small cell to another nearest small cell.

\subsection{Massive MIMO}

5G technology is intended to provide high data speed and low latency that can be covered by the high-power transmission but due to high power, radiation effect will be caused that may be hazardous to human health. Hence different types of diversity techniques are utilized in 5G technology with different designing aspect with respect to current $4 \mathrm{G}$ system. The basic logic behind the space diversity is that, it uses multiple antennas for transmission and reception which will reduce bit error rate so the speed will automatically be increased [5,6].

5G technology also uses the MIMO technique for high-speed data. MIMO (multiple input, multiple output) is an antenna technology used for wireless communications in which multiple antennas are used at both the source (transmitter) and the destination (receiver). The antennas at each end of the communication circuit are combined to minimize errors and optimize data speed as shown in Fig. 1.

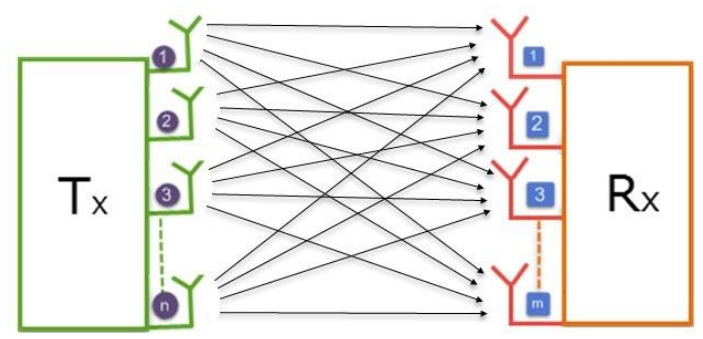

Fig. 1. Massive MIMO implementation for antenna diversity realization.

The transmitter and receiver shown in above Fig. are configured by using pair of source-sink, pair of encoder-decoder, pair of modulator-demodulator as well as pair of multiplexer-demultiplexer. Finally, the processed signals are applied to massive MIMO logic and thereby will be transmitted through multiple antennas. On the receiver side, again multiple antennas are used to receive the multipath radio signals and then processed further. This can improve today's network capacity by factor of 24 or more. Further MIMO comes with its own drawback. MIMO antennas able to transmit information in all direction at ones and this entire signal get a serious interference.

\subsection{Beam forming}

In the earlier technology, sectored antennas were used rather than the directive antenna. In previous technology i.e., in $4 \mathrm{G}$, the power of the radiated antenna is high rather than the beam forming antenna which will be the key modification in antenna designing of $5 \mathrm{G}$ system. It can be clearly seen in Fig. 2 that there is sectored antenna whose radiation 
pattern is in all direction. In the previous and current wireless cellular technologies, these types of antennas are used.

Whereas in 5G technology the radiation pattern of antenna is directive as well as concentrated in a particular coverage area as shown in Fig. 3. So, the power level is low as compare to previous technology antennas. In $5 \mathrm{G}$, to decrease the latency of time every specific user with specific beam may be used. In this technique the focus of a wireless signal will be towards a specific receiving device, rather than having the signal spread in all directions from broadcast antenna. In beam forming, directive antenna will be used for the transmission of radio signals which would allow the base station to send focused data stream to specific user.

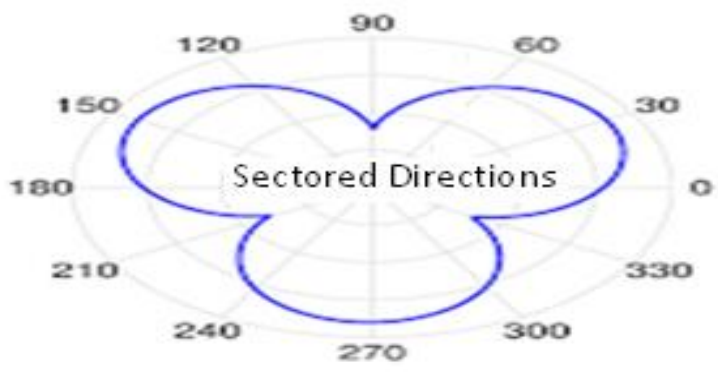

Fig. 2. Sectored antenna radiation pattern utilized in older and current cellular technology.

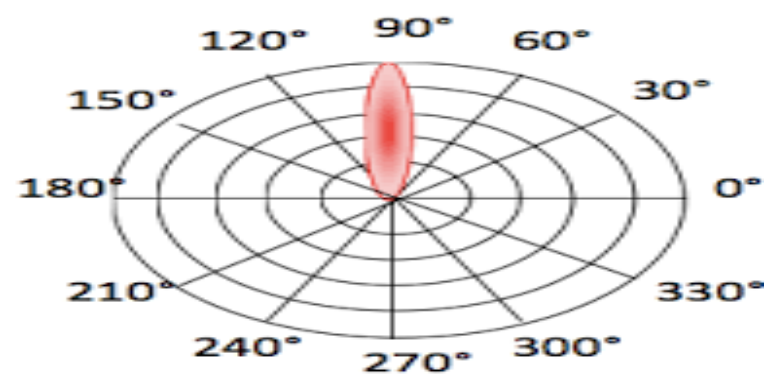

Fig. 3. Antenna radiation pattern with beam forming utilized in 5G technology.

On $23^{\text {rd }}$ February 2018, Bharti Airtel and Chinese multinational telecom gear Huawei have successfully conducted India's first 5G network trial under a test setup at the former's network experience center in Gurgaon. There are advantages of $5 \mathrm{G}$ for the developing country like India for their growing infrastructure in the IT technology. Important advantages of $5 \mathrm{G}$ are its high speed with high capacity, and low bit error rate. It supports high quality voice, video, Internet, and other broadband services, more effective and more attractive, and have bi-directional, accurate traffic statistics. It also offers Global 
access and service portability [7]. It is providing large broadcasting capacity up to Gigabit which is supporting almost 75,000 connections at a time. 5G will offer high resolution for crazy cell phone user and bi-directional large bandwidth shaping.

However apart from the above-mentioned utilities and benefits of $5 \mathrm{G}$ implementation, the system will also lead to certain types of cons related to livelihood health over the globe. Some of the health issues are discussed in the following section.

\section{Health Issues}

The majority of human exposure to ionizing radiation occurs from natural sources including cosmic rays and terrestrial radiation [8]. As discussed in previous sections that $5 \mathrm{G}$ technology is centered on high frequency spectrum i.e., millimeter wave spectrum, it is necessary to analyze the behavior of the same over human body. There are certain characteristics of $\mathrm{mm}$ wave signals that affect the human body parts like the millimeter wave can easily be absorbed by the human skin within 1 2 millimeter. The cornea which is the transparent part of the iris can also absorb the radiation. Nerve endings are connected with the skin to the radiation can reach our nervous and DNA system [9]. This section discusses in detail the negative effects of $\mathrm{mm}$ wave that are used in 5G transmission over different human body parts.

\subsection{Carcinogen properties of $5 G$ radiations}

In 2011 IARC had evaluated RF radiation as one of the possible human carcinogens. An IARC Monographs Working Group reviewed epidemiological evidence, cancer bioassays, and mechanistic and other relevant data to reach conclusions as to the carcinogenic hazard to humans from exposure to the electromagnetic fields that can occur from $2 \mathrm{G}, 3 \mathrm{G}, 4 \mathrm{G}$ handsets as well as from base station radiations [10]. Moreover, there is strong evidence that excessive exposure to mobile phone-frequencies over long periods of time increases the risk of brain cancer both in humans and animals [11]. However, as compare to older techniques, in $5 \mathrm{G}$, the utilization of high band spectrum, beam forming and MIMO techniques along with quite much level of radiation can mutate cell which will lead to cancer and tumor. Further the low intensity radio frequency radiations (RFR) create oxidative effects in living cells in terms of increased level of the reactive oxygen species (ROS) [12]. Reactive oxygen species are chemically reactive species containing oxygen and it is the part of signaling the cell. Overproduction of ROS that is not balanced with either endogenous antioxidants (superoxide dismutase (SOD), catalase (CAT), glutathione peroxidase (GPx), glutathione (GSH), melatonin), or exogenous antioxidants (Vitamin C, Vitamin E, carotenoids, polyphenols) allows the formation of free radicals that oxidize and damage DNA, proteins, membrane lipids and mitochondria as shown in Fig. 4 [10]. 


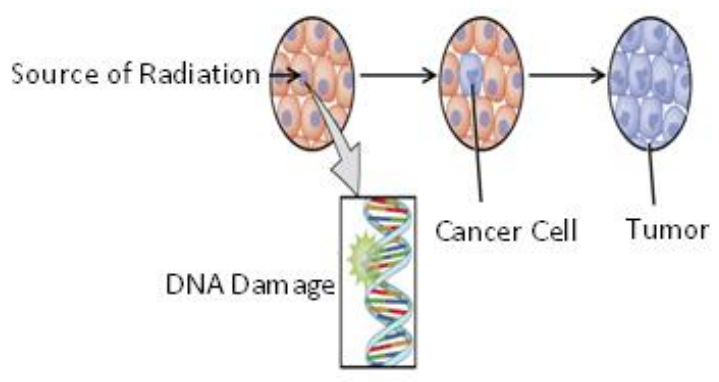

Fig. 4. Effect of radiation on Cell with damaged DNA and membrane lipids.

\subsection{Radiation effects on skin}

The radiation effects may heat the skin and the effects of heat generation may alter the temperature distribution [13]. 5G uses MIMO antennas along with beam forming technique wherein number of towers will get increase so the number of beams forming antennas pointing at certain user by means of highly directive beam may be hazardous.

Human skin consists of two layers-outer epidermis and an underlying dermis, with thicknesses varying in the range of 0.06 to $0.1 \mathrm{~mm}$ and 1.2 to $2.8 \mathrm{~mm}$, the relative permittivity of the skin decreases with the increase in the frequency whereas the conductivity will get increased.

Low-intensity millimeter microwave inducing a number of biological changes, even at non thermal levels, including cell membrane effects. Extreme broadband wireless devices operating above $10 \mathrm{GHz}$ may transmit data in bursts of a few milliseconds to seconds. Even though the time- and area-averaged power density values remain within the acceptable safety limits for continuous exposure, these bursts may lead to short temperature spikes in the skin of exposed people. Transient exposure with high PAR (peak to average power) can lead to large temperature oscillations, with peak temperature increases in the skin reaching tens of degrees, thus exceeding tissue damage thresholds after short exposure durations. Exposure to radiofrequency (RF) radiation from wireless devices to large radar installations and medical equipment can result in increases in body core temperature or cause localized temperature rises, with the potential for adverse health effects. The thresholds for frequencies above $10 \mathrm{MHz}$ set in current exposure guidelines (ICNIRP 1998; IEEE 2005, 2010) are intended to limit tissue heating. As exposure is frequently transient and pulsed, rather than continuous, limits are expressed in terms of time-averaged quantities. The International Council on Non-Ionizing Radiation Protection (ICNIRP) defines an averaging time of $68 \mathrm{fG}-1.05 \mathrm{~min}$ (where $\mathrm{fG}$ is the frequency in $\mathrm{GHz}$ ), while IEEE specifies $19.63 \mathrm{fG}-1.079 \mathrm{~min}$ for frequencies below $30 \mathrm{GHz}$ and $2.524 \mathrm{fG}-0.476 \mathrm{~min}$ for those above. The averaging durations have been derived considering the time constant of in vivo temperature evolution. An example of the transient temperature during a single oscillation period is shown in Fig. 5. 


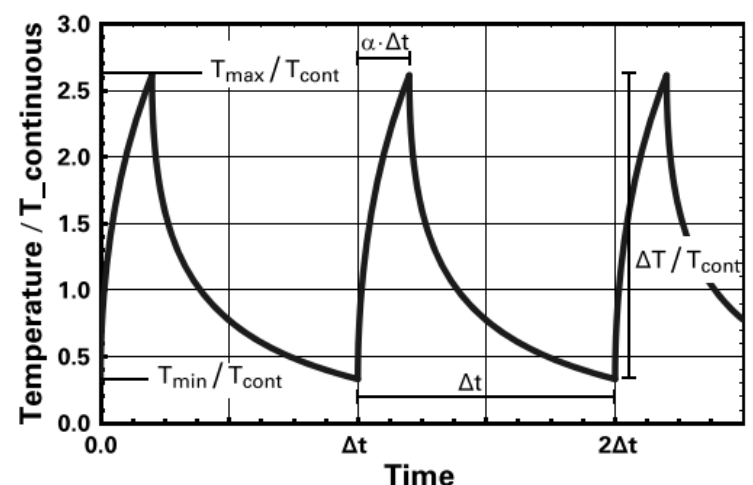

Fig. 5. Transient temperature oscillations resulting from a pulse train resulting in temperature increase of $1 \mathrm{~K}$ with continuous exposure [14].

However, short pulses can lead to important temperature oscillations, which may be further exacerbated at high frequencies (>10 GHz, fundamental to $5 \mathrm{G}$ ), where the shallow penetration depth leads to intense surface heating and a steep, rapid rise in temperature (e.g., proportional to sqrt(t) for plane-wave exposure [14].

\subsection{Effect on eyes}

Due to massive MIMO technique and beam forming technique, radiation effects are quite greater than old technology but the power of the transmitting signal is low as compared to old technology [15].

A cataract is a clouding of the lens of the eye which leads to a decrease in vision. In the near field exposure of $5 \mathrm{G}$ antenna, the tissues of eye will be in direct contact with the radiation. So, the chances of cataract will get increased. Microwave radiation is also a known cause of cataracts with heat being an undisputed mechanism. The eyes lack sufficient blood flow to dissipate heat effectively. There is some evidence that repeated low-level exposures to microwave radiation could cause cataracts as shown in Fig. 6.

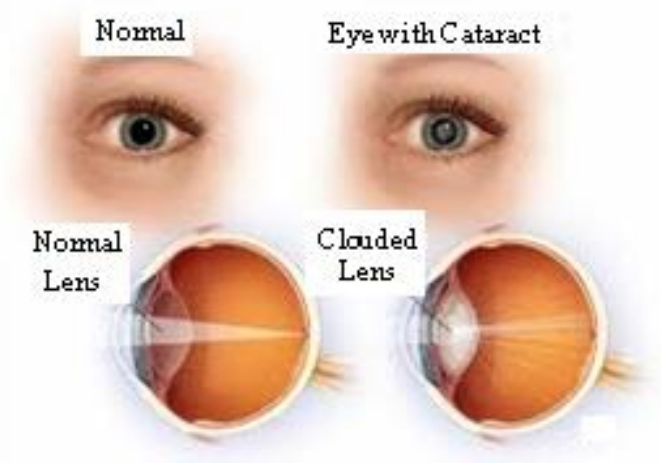

Fig. 6. Effect of radiation in eyes. 
Other issues from electromagnetic sensitivity are headaches, insomnia, dizziness, nausea, lack of concentration, heart palpitations, and depression.

\subsection{Neurological effects}

When the nervous system or the brain is disturbed by EMR, morphological, electrophysiological, and chemical changes can occur. A significant change in these functions will inevitably lead to a change in behavior.

The neurological effects of EMR reported in the literature include changes in blood brain-barrier, morphology, electrophysiology, neurotransmitter functions, cellular metabolism, calcium efflux, responses to drugs that affect the nervous system [15].

\subsection{Thermal effects}

The receiving mobile phone have single receiving antenna but the number of transmitting antenna will be more so the electromagnetic effect will be more as compare to old technology [15].

When EM radiation is absorbed, it is converted into heat. A readily understandable mechanism of the effect of radiation is tissue heating (thermal effect). Biological systems alter their functions as a result of a change in temperature. When electromagnetic radiation is incident on the matter, it causes the charged particles to oscillate and gain energy. The ultimate fate of this energy depends on the situation. It could be immediately re-radiated and appear as scattered, reflected, or transmitted radiation as shown in Fig. 7 [16].

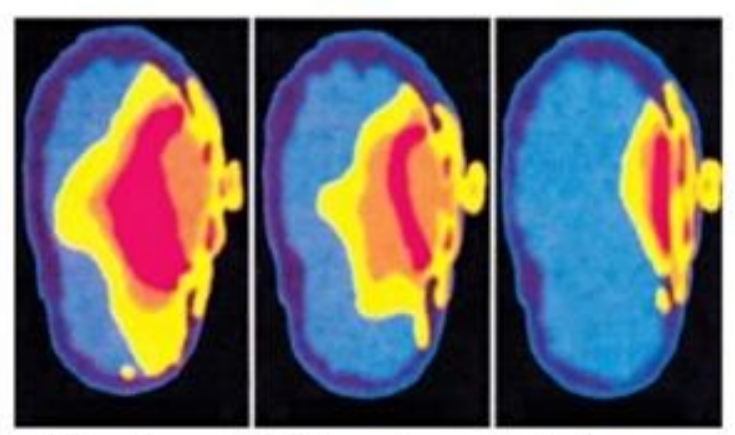

5 Year Person 10 Year Person Adult Person

Fig. 7. Thermal effect of tissue heating.

\subsection{Non-thermal effects}

In 2011, the International Agency for Research on Cancer (IARC) reviewed the published literature and categorized RFR as a "possible" (Group 2B) human carcinogen. A broad range of adverse human health effects associated with RFR has been reported since the 
IARC review [17]. In a study of four groups of men, of which one group did not use mobile phones, it was found that DNA damage indicators in hair follicle cells in the ear canal were higher in the RFR exposure groups than in the control subjects. In addition, DNA damage increased with the daily duration of exposure [18]. As a result of rapid growth rates and the greater vulnerability of developing nervous systems, the long-term risks to children from RFR exposure from cell phones and other WTDs are expected to be greater than those to adults [19]. By analogy with other carcinogens, longer opportunities for exposure due to earlier use of cell phones and other WTDs could be associated with greater cancer risks in later life.

An extensive review of numerous published studies confirms non-thermally induced biological effects or damage (e.g., oxidative stress, damaged DNA, gene and protein expression, breakdown of the blood-brain barrier) from exposure to RFR [20], as well as adverse (chronic) health effects from long-term exposure.

\section{Conclusion}

This analytical paper totally declines the possibility of spreading of the Covid-19 through the $5 \mathrm{G}$ technology antenna. There will be health hazard due to beam forming and massive MIMO techniques but it will decrease the immune system of human body over the long time. Short term immunity reduction will not at all anticipated by any of the technique used for the realization of $5 \mathrm{G}$ wireless cellular system.

As a whole some of the health issues may take place because of the excess usage of $5 \mathrm{G}$ over a period of long time but Covid-19 is not at all the byproduct of $5 \mathrm{G}$ cellular implementation. Infect on the other side of coin that is the brighter side of $5 \mathrm{G}$ system realization ensures very high speed and efficient data communication that will make real time data processing very effective and easy.

\section{References}

1. I. Belyaev, Main Regularities and Health Risks from Exposure to Non-Thermal Microwaves of Mobile Communication - IEEE Transactions on Communications, TELSIKS (2020). https://doi.org/10.1109/TELSIKS46999.2019.9002324

2. A. W. Scott, R. Frobenius, and R. Frobenius, Measurements for Cellular Phones and Wireless Data Systems (Wiley- IEEE Press, 2008). https://doi.org/10.1002/9780470378014

3. L. Zhang, A. Ijaz, P. Xiao, M. Molu, and R. Tafazolli, IEEE Transact. Commun. 66, 1205 (2018). https://doi.org/10.1109/TCOMM.2017.2771242

4. J. G. Andrews, S. Buzzi, W. Choi, S. V. Hanly, A. Lozano, A. C. K.Soong, and J. C. Zhang, IEEE J. Sel. Areas Commun. 32, 1065 (2014). https://doi.org/10.1109/JSAC.2014.2328098

5. C. -L. I. C. Rowell, S. Han, Z. Xu, G. Li, and Z. Pan, IEEE Commun. Mag. 52, 66 (2014). https://doi.org/10.1109/MCOM.2014.6736745

6. F. Boccardi, R. W. Heath Jr., A. Lozano, T. L. Marzetta, and P. Popovski, IEEE Commun. Mag. 52, 74 (2014). https://doi.org/10.1109/MCOM.2014.6736746

7. B. Bangerter, S. Talwar, R. Arefi, and K. Stewart, IEEE Commun. Mag. 52, 90 (2014). https://doi.org/10.1109/MCOM.2014.6736748

8. M. S. Mian, M. S. Rahman, J. Islam, K. N. Sakib, M. M. Tasnim, and S. Yeasmin, J. Sci. Res. 11, 263 (2019). https://doi.org/10.3329/jsr.v11i3.39318 
9. I. Belyaev, Microw. Rev. 11, 13 (2005)

10. IARC Working Group on the Evaluation of Carcinogenic Risks to Humans, IARC Monogr. Eval. Carcinog. Risks Hum. 102, 1 (2013).

11. D. Belpomme, L. Hardell, I. Belyaev, E. Burgio, and D. O. Carpenter, Environ. Pollut. 242, 643 (2018). https://doi.org/10.1016/j.envpol.2018.07.019

12. I. Yakymenko, O. Tsybulin, E. Sidorik, D. Henshel, O. Kyrylenko, and S. Kyrylenko, Electromagn. Biol. Med. 35, 186 (2016). https://doi.org/10.3109/15368378.2015.1043557

13. M. Y. Ali, N. M. R. Zahed, M. N. Uddin, and M. J. Uddin, J. Sci. Res. 8, 341 (2016). https://doi.org/10.3329/jsr.v8i3.27851

14. O. P. Gandhi, L. L. Morgan, A. A. d. Salles, Y.-Y. Han, R. B. Herberman, and D. L. Davis, Electromag. Biolo. Med. 31, 34 (2011). https://doi.org/10.3109/15368378.2011.622827

15. C. Verma, T. M. Tejaswini, and D. Pradhan, Harmful Effects of 5G Radiations: Review Proc. of IRAJ Int. Conf. (2019).

16. E. Neufeld and N. Kuster, Health Phys. 115, 705 (2018). https://doi.org/10.1097/HP.0000000000000930

17. A. B. Miller, M. E. Sears, L. L. Morgan, D. L. Davis, L. Hardell, M. Oremus, and C. L. Soskolne, Front. Public Health. 7, 223 (2019). https://doi.org/10.3389/fpubh.2019.00223

18. M. Akdag, S. Dasdag, F. Canturk, and M. Z. Akdag, Electromag. Biol. Med. 37, 66 (2018). https://doi.org/10.1080/15368378.2018.1463246

19. M. Redmayne, E. Smith, and M. J. Abramson, Environ. Health 12, ID 90 (2013). https://doi.org/10.1186/1476-069X-12-90

20. BioInitiative Working Group, A Rationale for Biologically-Based Exposure Standards for Low-Intensity Electromagnetic Radiation (2012). https://www.bioinitiative.org/

21. N. Bhushan, J. Li, D. Malladi, R. Gilmore, D. Brenner, A. Damnjanovic, R. T. Sukhavasi, C. Patel, and S. Geirhofer, IEEE Commun. Mag. 52, 82 (2014). https://doi.org/10.1109/MCOM.2014.6736747

22. Chronic Disease Prevention and Health Promotion, CDC (2017). 